Article

\title{
Analytical Solution of Nonlinear Fractional Volterra Population Growth Model Using the Modified Residual Power Series Method
}

\author{
Patcharee Dunnimit ${ }^{1}$, Araya Wiwatwanich ${ }^{1}$ (D) and Duangkamol Poltem ${ }^{1,2, * \mathbb{D}}$ \\ 1 Department of Mathematics, Faculty of Science, Burapha University, Chonburi 20131, Thailand; \\ 61910071@go.buu.ac.th (P.D.); arayawi@go.buu.ac.th (A.W.) \\ 2 Centre of Excellence in Mathematics, Commission on Higher Education, Ministry of Education, \\ Bangkok 10400, Thailand \\ * Correspondence: duangkamolp@buu.ac.th; Tel.: +6-638-103-099
}

Received: 1 October 2020; Accepted: 23 October 2020; Published: 27 October 2020

\begin{abstract}
In this paper, we introduce an analytical approximate solution of nonlinear fractional Volterra population growth model based on the Caputo fractional derivative and the Riemann fractional integral of the symmetry order. The residual power series method and Adomain decomposition method are implemented to find an approximate solution of this problem. The convergence analysis of the proposed technique has been proved. A numerical example is given to illustrate the method.
\end{abstract}

Keywords: fractional differential equations; population growth model; fractional power series; residual power series method

\section{Introduction}

In recent years, fractional calculus appears frequently in the context of mathematical modeling in various branches of science and engineering such as robotics [1], control theory [2], signal processing [3], economics [4], viscoelasticity [5]. For more details and applications about fractional calculus, we refer the reader to [6-9]. In most cases, the exact solutions for fractional nonlinear problems, if exist, are not easy to find [10-13]. In order to describe the behavior of the unknowns of those systems, many researchers usually perform some numerical or approximate analytical methods instead. In this regard, some recent techniques are proposed for solving fractional functional equations. Among them are sorts of integral transform methods which are well combined with the homotopy analysis methods [14-19]. The Adomian decomposition method (ADM) [20] and the variational iteration method [21,22] are also mentioned in many contexts. The residual power series method (RPSM) is one of those techniques which quite suits nonlinear fractional differential equations [23-30]. Generalized from the classical power series method, the solution is written on the form of fractional power series. However, the formula of all coefficients can be derived by enormous algebraic manipulations. The main merit of the RPSM is that the series solution, in particular a truncated series solution can be easily obtained.

The study of population growth model is one of the specific fields of science which is gaining attention due to the limitation of resources on our planet. The Volterra model for population growth [31] in a closed system is represented by the nonlinear Volterra integro-differential equation

$$
\begin{aligned}
& \kappa \frac{d u}{d t}=u-u^{2}-u \int_{0}^{t} u(\tau) d \tau, \\
& u(0)=a_{0}, \quad a_{0}>0,
\end{aligned}
$$


where $u \equiv u(t)$ is the scaled population of identical individuals at a time $t$ and $a_{0}$ is an initial population. The nondimensional parameter $\kappa=\frac{c}{a b}$ is introduced to explain overall increasing or decreasing rate of the population, where $a>0, b>0$ and $c>0$ denote the birth rate coefficient, the crowding coefficient and the toxicity coefficient, respectively [32].

In this study, we consider the following nonlinear fractional Volterra population growth model of the form:

$$
\kappa D_{t}^{\alpha} u(t)=u(t)-u^{2}(t)-u(t) I^{\alpha} u(t), \alpha \in(0,1],
$$

subject to

$$
u(0)=a_{0}, \quad a_{0}>0 .
$$

The derivative in the fractional Volterra population growth model (3) is in the Caputo sense and $I^{\alpha}$ is the Riemann-Liouville fractional integral operator of order $\alpha$. In order to keep the symmetry aspect, we set the order of the derivative and the integral to be equal. Due to the nonlinear term in Equation (3), solving this problem by RPSM is most likely cumbersome. In various method mentioned above the well-known Adomian polynomials take their part to handle this difficulty. Actually, the Adomian polynomials was introduced by George Adomian in 1988 [33] as a sequence of series of Maclaurin type embedded in the ADM for nonlinear problems. The ADM has become a powerful technique for analytic approximate solutions to initial value problems. Furthermore, according to [34-36] the Adomian polynomials and the ADM itself can be combined well with other methods.

Motivated by the existing methods, the main objective of this paper is to study the nonlinear fractional Volterra population growth model using the residual power series method and the Adomian decomposition method. This method is called modified residual power series method (MRPSM). The remaining sections of this paper are organized as follows. In Section 2, we present some preliminaries of fractional calculus and the fractional power series. Applications of the MRPSM to the nonlinear fractional Volterra population growth model are presented in Section 3. In Section 4, the convergence analysis is investigated. In Section 5, the graphical result is also reported for different values of fractional parameter. Finally, in Section 6 some conclusions are drawn.

\section{Preliminaries}

In this section, we give some preliminaries of fractional calculus and fractional power series [37,38], which are further used in this paper.

Definition 1. Let $u(t) \in C^{n}(0, \infty)$. The Caputo fractional derivative of order $\alpha>0$ is defined as

$$
D_{t}^{\alpha} u(t)= \begin{cases}\frac{1}{\Gamma(n-\alpha)} \int_{0}^{t}(t-\tau)^{n-\alpha-1} u^{(n)}(\tau) d \tau, & n-1<\alpha<n, \\ u^{(n)}(t), & \alpha=n \in \mathbb{N},\end{cases}
$$

where $n$ is the smallest integer greater than or equal to $\alpha$ and $\Gamma(\cdot)$ is the well-known Gamma function.

Theorem 1. The Caputo fractional derivative of the power function is as follows

$$
D_{t}^{\alpha} t^{p}= \begin{cases}\frac{\Gamma(p+1)}{\Gamma(p-\alpha+1)} t^{p-\alpha}, & n-1<\alpha<n, p>n-1, p \in \mathbb{R} \\ 0, & n-1<\alpha<n, p \leq n-1, p \in \mathbb{N} .\end{cases}
$$

Definition 2. The Riemann-Liouville fractional integral operator $I^{\alpha}$ of order $\alpha>0$ is normally defined by

$$
I^{\alpha} u(t)= \begin{cases}\frac{1}{\Gamma(\alpha)} \int_{0}^{t} \frac{u(\tau)}{(t-\tau)^{1-\alpha}} d \tau, & \alpha>0, \\ u(t), & \alpha=0 .\end{cases}
$$

where $u(t)$ is a function defined on $(0, t]$. 
Theorem 2. The Riemann-Liouville fractional integral operator of power function is given by

$$
I^{\alpha} t^{p}=\frac{\Gamma(p+1)}{\Gamma(p+\alpha+1)} t^{p+\alpha} .
$$

The following definition and theorem related to the RPSM [38].

Definition 3. The fractional power series (FPS) about $t=t_{0}$ is given by

$$
\sum_{m=0}^{\infty} c_{m}\left(t-t_{0}\right)^{m \alpha}=c_{0}+c_{1}\left(t-t_{0}\right)^{\alpha}+c_{2}\left(t-t_{0}\right)^{2 \alpha}+\cdots,
$$

where $0 \leq n-1<\alpha$ and $t \geq t_{0}$.

Theorem 3. Suppose that $f$ has a fractional power series represent at $t=t_{0}$ of the form

$$
f(t)=\sum_{m=0}^{\infty} c_{m}\left(t-t_{0}\right)^{m \alpha},
$$

where $0 \leq n-1<\alpha, t_{0} \leq t<t_{0}+R$ and $R$ is the radius of convergence.

If $D^{m \alpha} f(t), m=0,1,2, \ldots$ are continuous on $\left(t_{0}, t_{0}+R\right)$, then $c_{m}=\frac{D^{m \alpha} f\left(t_{0}\right)}{\Gamma(1+m \alpha)}$.

\section{Modified Residual Power Series Method (MRPSM) for Nonlinear Fractional Volterra Population Growth Model}

Consider the fractional nonlinear Volterra population growth model

$$
\kappa D_{t}^{\alpha} u(t)=u(t)-u^{2}(t)-u(t) I^{\alpha} u(t), \alpha \in(0,1],
$$

subject to

$$
u(0)=a_{0}, a_{0}>0,
$$

where $\kappa>0$ is a prescribed non-dimensional parameter and $u(t)$ is the scaled population of identical individuals at time $t$. The derivative in the fractional Volterra population growth model (5) is in the Caputo sense and $I^{\alpha}$ is the Riemann-Liouville fractional integral operator of order $\alpha$.

According to the RPSM, let $u(t)$ be the solution of fractional Volterra population growth model of the form:

$$
u(t)=\sum_{n=0}^{\infty} \frac{a_{n} t^{n \alpha}}{\Gamma(1+n \alpha)} .
$$

Using the initial condition (6), we approximate $u(t)$ in Equation (7) by

$$
u_{k}(t)=a_{0}+\sum_{n=1}^{k} \frac{a_{n} t^{n \alpha}}{\Gamma(1+n \alpha)}, k=1,2,3, \ldots
$$

To find the residual power series (RPS) coefficient $a_{n}$, we solve the equation

$$
D_{t}^{(n-1) \alpha} \operatorname{Res}_{n}(0)=0, n=1,2,3, \ldots,
$$

where $\operatorname{Res}_{k}(t)$ is the $k$ th residual function and is defined by

$$
\operatorname{Res}_{k}(t)=\kappa D_{t}^{\alpha} u_{k}(t)-u_{k}(t)+u_{k}^{2}(t)+u_{k}(t) I^{\alpha} u_{k}(t) .
$$

Since the fractional Volterra population growth model (5) has a nonlinear term $F(u)=u^{2}(t)$, the Adomian polynomials play their role in dealing with it. 
Let

$$
u_{k}(t)=\sum_{i=0}^{k} v_{i}
$$

where $v_{0}=a_{0}$ and

$$
v_{i}=\frac{a_{i} t^{i \alpha}}{\Gamma(1+i \alpha)}, i=1,2,3, \ldots, k
$$

Let

$$
F\left(u_{k}(t)\right)=\sum_{n=0}^{k} A_{n}
$$

be a nonlinear operator where $A_{n}$ are called Adomian polynomials and can be determined from the formula

$$
A_{n}=\frac{1}{n !}\left[\frac{d^{n}}{d \lambda^{n}}\left[F\left(\sum_{i=0}^{n} \lambda^{i} v_{i}\right)\right]\right]_{\lambda=0} .
$$

From Equation (11), we can rewritten the nonlinear polynomials $u_{k}^{2}(t)$ as

$$
F\left(u_{k}(t)\right)=\left(v_{0}+v_{1}+v_{2}+v_{3}+\cdots+v_{k}\right)^{2}=\sum_{n=0}^{k} A_{n} .
$$

Using the Algorithm presented in [39], the Adomian polynomials for $F\left(u_{k}(t)\right)=u_{k}^{2}(t)$ are given by

$$
\begin{aligned}
& A_{0}=v_{0}^{2} \\
& A_{1}=2 v_{0} v_{1} \\
& A_{2}=2 v_{0} v_{2}+v_{1}^{2} \\
& A_{3}=2 v_{0} v_{3}+2 v_{1} v_{2} \\
& A_{4}=v_{2}^{2}+2 v_{1} v_{3}+2 v_{0} v_{4} \\
& A_{5}=2 v_{2} v_{3}+2 v_{0} v_{5}+2 v_{1} v_{4} \\
& A_{6}=2 v_{0} v_{6}+2 v_{1} v_{5}+2 v_{2} v_{4}+v_{3}^{2} \\
& A_{7}=2 v_{0} v_{7}+2 v_{2} v_{5}+2 v_{3} v_{4}+2 v_{1} v_{6} \\
& A_{8}=2 v_{2} v_{6}+2 v_{3} v_{5}+v_{4}^{2}+2 v_{0} v_{8}+2 v_{1} v_{7} .
\end{aligned}
$$

Other polynomials can be calculated by Equation (14).

To find $a_{1}$, we substitute the first RPS approximate solution

$$
u_{1}(t)=a_{0}+a_{1} \frac{t^{\alpha}}{\Gamma(1+\alpha)}
$$

into Equation (10) as follows

$$
\begin{aligned}
\operatorname{Res}_{1}(t)= & \kappa D_{t}^{\alpha} u_{1}(t)-u_{1}(t)+u_{1}^{2}(t)+u_{1}(t) I^{\alpha}\left(u_{1}(t)\right) \\
= & \kappa D_{t}^{\alpha}\left(a_{0}+a_{1} \frac{t^{\alpha}}{\Gamma(1+\alpha)}\right)-\left(a_{0}+a_{1} \frac{t^{\alpha}}{\Gamma(1+\alpha)}\right) \\
& +\left(a_{0}+a_{1} \frac{t^{\alpha}}{\Gamma(1+\alpha)}\right)^{2}+\left(a_{0}+a_{1} \frac{t^{\alpha}}{\Gamma(1+\alpha)}\right) I^{\alpha}\left(a_{0}+a_{1} \frac{t^{\alpha}}{\Gamma(1+\alpha)}\right) \\
= & \kappa a_{1}-\left(a_{0}+a_{1} \frac{t^{\alpha}}{\Gamma(1+\alpha)}\right)+\left(a_{0}+a_{1} \frac{t^{\alpha}}{\Gamma(1+\alpha)}\right)^{2} \\
& +\left(a_{0}+a_{1} \frac{t^{\alpha}}{\Gamma(1+\alpha)}\right)\left(a_{0} \frac{t^{\alpha}}{\Gamma(1+\alpha)}+a_{1} \frac{t^{2 \alpha}}{\Gamma(1+2 \alpha)}\right) .
\end{aligned}
$$


Then, we solve $\operatorname{Res}_{1}(0)=0$ to get

$$
a_{1}=\frac{1}{\kappa}\left[a_{0}-a_{0}^{2}\right] .
$$

To find $a_{2}$, the second RPS approximate solution is in form

$$
u_{2}(t)=a_{0}+a_{1} \frac{t^{\alpha}}{\Gamma(1+\alpha)}+a_{2} \frac{t^{2 \alpha}}{\Gamma(1+2 \alpha)} .
$$

Using Adomian polynomials for $F\left(u_{2}(t)\right)=u_{2}^{2}(t)$ and $v_{0}=a_{0}$, we have

$$
\begin{aligned}
u_{2}^{2}(t)= & a_{0}^{2}+2 a_{0} a_{1} \frac{t^{\alpha}}{\Gamma(1+\alpha)}+2 a_{0} a_{2} \frac{t^{2 \alpha}}{\Gamma(1+2 \alpha)}+\left(a_{1} \frac{t^{\alpha}}{\Gamma(1+\alpha)}\right)^{2} \\
& +2 a_{1} a_{2} \frac{t^{3 \alpha}}{\Gamma(1+\alpha) \Gamma(1+2 \alpha)}+\left(a_{2} \frac{t^{2 \alpha}}{\Gamma(1+2 \alpha)}\right)^{2} .
\end{aligned}
$$

On substituting Equations (16) and (17) in Equation (10), we have

$$
\begin{aligned}
\operatorname{Res}_{2}(t)= & \kappa D_{t}^{\alpha} u_{2}(t)-u_{2}(t)+u_{2}^{2}(t)+u_{2}(t) I^{\alpha}\left(u_{2}(t)\right) \\
= & \kappa\left(a_{1}+a_{2} \frac{t^{\alpha}}{\Gamma(1+\alpha)}\right)-\left(a_{0}+a_{1} \frac{t^{\alpha}}{\Gamma(1+\alpha)}+a_{2} \frac{t^{2 \alpha}}{\Gamma(1+2 \alpha)}\right) \\
& +\left[a_{0}^{2}+2 a_{0} a_{1} \frac{t^{\alpha}}{\Gamma(1+\alpha)}+2 a_{0} a_{2} \frac{t^{2 \alpha}}{\Gamma(1+2 \alpha)}+\left(a_{1} \frac{t^{\alpha}}{\Gamma(1+\alpha)}\right)^{2}\right. \\
& \left.+2 a_{1} a_{2} \frac{t^{3 \alpha}}{\Gamma(1+\alpha) \Gamma(1+2 \alpha)}+\left(a_{2} \frac{t^{2 \alpha}}{\Gamma(1+2 \alpha)}\right)^{2}\right] \\
& +\left[\left(\frac{a_{0}^{2}}{\Gamma(1+\alpha)}\right) t^{\alpha}+\left(\frac{a_{0} a_{1}}{\Gamma^{2}(1+\alpha)}+\frac{a_{0} a_{1}}{\Gamma(1+2 \alpha)}\right) t^{2 \alpha}\right. \\
& +\left(\frac{a_{0} a_{2}}{\Gamma(1+\alpha) \Gamma(1+2 \alpha)}+\frac{a_{1}^{2}}{\Gamma(1+\alpha) \Gamma(1+2 \alpha)}+\frac{a_{0} a_{2}}{\Gamma(1+3 \alpha)}\right) t^{3 \alpha} \\
& \left.+\left(\frac{a_{1} a_{2}}{\Gamma^{2}(1+2 \alpha)}+\frac{a_{1} a_{2}}{\Gamma(1+\alpha) \Gamma(1+3 \alpha)}\right) t^{4 \alpha}+\left(\frac{a_{2}^{2}}{\Gamma(1+2 \alpha) \Gamma(1+3 \alpha)}\right) t^{5 \alpha}\right] .
\end{aligned}
$$

On applying the derivative $D_{t}^{\alpha}$ on Equation (18), we obtain

$$
\begin{aligned}
D_{t}^{\alpha} \operatorname{Res}_{2}(t)= & \kappa a_{2}-\left(a_{1}+a_{2} \frac{t^{\alpha}}{\Gamma(1+\alpha)}\right) \\
& +\left[2 a_{0} a_{1}+2 a_{0} a_{2} \frac{t^{\alpha}}{\Gamma(1+\alpha)}+a_{1}^{2} \frac{\Gamma(1+2 \alpha) t^{\alpha}}{\Gamma^{3}(1+\alpha)}\right. \\
& \left.+2 a_{1} a_{2} \frac{\Gamma(1+3 \alpha) t^{2 \alpha}}{\Gamma(1+\alpha) \Gamma^{2}(1+2 \alpha)}+a_{2}^{2} \frac{\Gamma(1+4 \alpha) t^{3 \alpha}}{\Gamma^{2}(1+2 \alpha) \Gamma(1+3 \alpha)}\right] \\
& +\left[a_{0}^{2}+\left(\frac{a_{0} a_{1}}{\Gamma^{2}(1+\alpha)}+\frac{a_{0} a_{1}}{\Gamma(1+2 \alpha)}\right) \frac{\Gamma(1+2 \alpha) t^{\alpha}}{\Gamma(1+\alpha)}\right. \\
& +\left(\frac{a_{0} a_{2}}{\Gamma(1+\alpha) \Gamma(1+2 \alpha)}+\frac{a_{1}^{2}}{\Gamma(1+\alpha) \Gamma(1+2 \alpha)}+\frac{a_{0} a_{2}}{\Gamma(1+3 \alpha)}\right) \frac{\Gamma(1+3 \alpha) t^{2 \alpha}}{\Gamma(1+2 \alpha)} \\
& \left.+\left(\frac{a_{1} a_{2}}{\Gamma^{2}(1+2 \alpha)}+\frac{a_{1} a_{2}}{\Gamma(1+\alpha) \Gamma(1+3 \alpha)}\right) \frac{\Gamma(1+4 \alpha) t^{3 \alpha}}{\Gamma(1+3 \alpha)}+\left(\frac{a_{2}^{2}}{\Gamma(1+2 \alpha) \Gamma(1+3 \alpha)}\right) \frac{\Gamma(1+5 \alpha) t^{4 \alpha}}{\Gamma(1+4 \alpha)}\right] .
\end{aligned}
$$

Then, we solve $D_{t}^{\alpha} \operatorname{Res}_{2}(0)=0$ to get

$$
a_{2}=\frac{1}{\mathcal{K}}\left[a_{1}-2 a_{0} a_{1}-a_{0}^{2}\right] .
$$

To find $a_{3}$, the third RPS approximate solution is in form

$$
u_{3}(t)=a_{0}+a_{1} \frac{t^{\alpha}}{\Gamma(1+\alpha)}+a_{2} \frac{t^{2 \alpha}}{\Gamma(1+2 \alpha)}+a_{3} \frac{t^{3 \alpha}}{\Gamma(1+3 \alpha)} .
$$


Using Adomian polynomials and $F\left(u_{3}(t)\right)=u_{3}^{2}(t)$, we have

$$
\begin{aligned}
u_{3}^{2}= & a_{0}^{2}+2 a_{0} a_{1} \frac{t^{\alpha}}{\Gamma(1+\alpha)}+2 a_{0} a_{2} \frac{t^{2 \alpha}}{\Gamma(1+2 \alpha)}+\left(a_{1} \frac{t^{\alpha}}{\Gamma(1+\alpha)}\right)^{2} \\
& +2 a_{0} a_{3} \frac{t^{3 \alpha}}{\Gamma(1+3 \alpha)}+2 a_{1} a_{2} \frac{t^{3 \alpha}}{\Gamma(1+\alpha) \Gamma(1+2 \alpha)}+\left(a_{2} \frac{t^{2 \alpha}}{\Gamma(1+2 \alpha)}\right)^{2} \\
& +2 a_{1} a_{3} \frac{t^{4 \alpha}}{\Gamma(1+\alpha) \Gamma(1+3 \alpha)}+2 a_{2} a_{3} \frac{t^{5 \alpha}}{\Gamma(1+2 \alpha) \Gamma(1+3 \alpha)}+\left(a_{3} \frac{t^{3 \alpha}}{\Gamma(1+3 \alpha)}\right)^{2} .
\end{aligned}
$$

On substituting Equations (20) and (21) in Equation (10), we obtain

$$
\begin{aligned}
\operatorname{Res}_{3}(t)= & \kappa D_{t}^{\alpha} u_{3}(t)-u_{3}(t)+u_{3}^{2}(t)+u_{3}(t) I^{\alpha}\left(u_{3}(t)\right) \\
= & \kappa\left(a_{1}+a_{2} \frac{t^{\alpha}}{\Gamma(1+\alpha)}+a_{3} \frac{t^{2 \alpha}}{\Gamma(1+2 \alpha)}\right) \\
& -\left(a_{0}+a_{1} \frac{t^{\alpha}}{\Gamma(1+\alpha)}+a_{2} \frac{t^{2 \alpha}}{\Gamma(1+2 \alpha)}+a_{3} \frac{t^{3 \alpha}}{\Gamma(1+3 \alpha)}\right) \\
& +\left(a_{0}^{2}+2 a_{0} a_{1} \frac{t^{\alpha}}{\Gamma(1+\alpha)}+2 a_{0} a_{2} \frac{t^{2 \alpha}}{\Gamma(1+2 \alpha)}+\left(a_{1} \frac{t^{\alpha}}{\Gamma(1+\alpha)}\right)^{2}\right. \\
& +2 a_{0} a_{3} \frac{t^{3 \alpha}}{\Gamma(1+3 \alpha)}+2 a_{1} a_{2} \frac{t^{3 \alpha}}{\Gamma(1+\alpha) \Gamma(1+2 \alpha)}+\left(a_{2} \frac{t^{2 \alpha}}{\Gamma(1+2 \alpha)}\right)^{2} \\
& \left.+2 a_{1} a_{3} \frac{t^{4 \alpha}}{\Gamma(1+\alpha) \Gamma(1+3 \alpha)}+2 a_{2} a_{3} \frac{t^{5 \alpha}}{\Gamma(1+2 \alpha) \Gamma(1+3 \alpha)}+\left(a_{3} \frac{t^{3 \alpha}}{\Gamma(1+3 \alpha)}\right)^{2}\right] \\
& +\left[\left(\frac{a_{0}^{2}}{\Gamma(1+\alpha)}\right) t^{\alpha}+\left(\frac{a_{0} a_{1}}{\Gamma^{2}(1+\alpha)}+\frac{a_{0} a_{1}}{\Gamma(1+2 \alpha)}\right) t^{2 \alpha}\right. \\
& +\left(\frac{a_{0} a_{2}}{\Gamma(1+\alpha) \Gamma(1+2 \alpha)}+\frac{a_{1}^{2}}{\Gamma(1+\alpha) \Gamma(1+2 \alpha)}+\frac{a_{0} a_{2}}{\Gamma(1+3 \alpha)}\right) t^{3 \alpha} \\
& +\left(\frac{a_{0} a_{3}}{\Gamma(1+\alpha) \Gamma(1+3 \alpha)}+\frac{a_{1} a_{2}}{\Gamma^{2}(1+2 \alpha)}+\frac{a_{1} a_{2}}{\Gamma(1+\alpha) \Gamma(1+3 \alpha)}+\frac{a_{0} a_{3}}{\Gamma(1+4 \alpha)}\right) t^{4 \alpha} \\
& +\left(\frac{a_{1} a_{3}}{\Gamma(1+\alpha) \Gamma(1+4 \alpha)}+\frac{a_{2}^{2}}{\Gamma(1+2 \alpha) \Gamma(1+3 \alpha)}+\frac{a_{1} a_{3}}{\Gamma(1+2 \alpha) \Gamma(1+3 \alpha)}\right) t^{5 \alpha} \\
& \left.+\left(\frac{a_{2} a_{3}}{\Gamma(1+2 \alpha) \Gamma(1+4 \alpha)}+\frac{a_{2} a_{3}}{\Gamma^{2}(1+3 \alpha)}\right) t^{6 \alpha}+\left(\frac{a_{3}^{2}}{\Gamma(1+3 \alpha) \Gamma(1+4 \alpha)}\right) t^{7 \alpha}\right] .
\end{aligned}
$$

Then, we solve $D_{t}^{2 \alpha} \operatorname{Res}_{3}(0)=0$ to get

$$
a_{3}=\frac{1}{\mathcal{K}}\left[u_{2}-\left(2 a_{0} a_{2}+a_{1}^{2} \frac{\Gamma(1+2 \alpha)}{\Gamma^{2}(1+\alpha)}\right)-\left(\frac{a_{0} a_{1} \Gamma(1+2 \alpha)}{\Gamma^{2}(1+\alpha)}+a_{0} a_{1}\right)\right] .
$$

The same procedure is performed to obtain $u_{4}(t)$ as

$$
u_{4}(t)=a_{0}+a_{1} \frac{t^{\alpha}}{\Gamma(1+\alpha)}+a_{2} \frac{t^{2 \alpha}}{\Gamma(1+2 \alpha)}+a_{3} \frac{t^{3 \alpha}}{\Gamma(1+3 \alpha)}+a_{4} \frac{t^{4 \alpha}}{\Gamma(1+4 \alpha)},
$$

where the coefficient $a_{4}$ can be calculated by

$$
\begin{aligned}
a_{4}= & \frac{1}{\mathcal{K}}\left[a_{3}-\left(2 a_{0} a_{3}+2 a_{1} a_{2} \frac{\Gamma(1+3 \alpha)}{\Gamma(1+\alpha) \Gamma(1+2 \alpha)}\right)\right. \\
& \left.-\left(a_{0} a_{2}+\frac{a_{1}^{2} \Gamma(1+3 \alpha)}{\Gamma(1+\alpha) \Gamma(1+2 \alpha)}+\frac{a_{0} a_{2} \Gamma(1+3 \alpha)}{\Gamma(1+\alpha) \Gamma(1+2 \alpha)}\right)\right] .
\end{aligned}
$$

In general, for a positive integer $k$, the coefficient $a_{k}$ for the approximate solution $u_{k}(t)$ in Equation (8) is supposed to be

$$
\begin{aligned}
a_{k}= & \frac{1}{\mathcal{\kappa}}\left[a_{k-1}-\left(\sum_{i=0}^{k-1} \frac{a_{i} a_{k-1-i} \Gamma(1+(k-1) \alpha)}{\Gamma(1+i \alpha) \Gamma(1+(k-1-i) \alpha)}\right)\right. \\
& \left.-\left(\sum_{i=0}^{k-2} \frac{a_{i} a_{k-2-i} \Gamma(1+(k-1) \alpha)}{\Gamma(1+i \alpha) \Gamma(1+(k-1-i) \alpha)}\right)\right] .
\end{aligned}
$$

We give the proof in the following theorem. 
Theorem 4. The nonlinear fractional Volterra population growth model (5) subject to the initial condition (6) has the approximate solution in the form

$$
u_{k}(t)=a_{0}+\sum_{n=1}^{k} \frac{a_{n} t^{n \alpha}}{\Gamma(1+n \alpha)}
$$

where

$$
\begin{aligned}
a_{k}= & \frac{1}{\mathcal{\kappa}}\left[a_{k-1}-\left(\sum_{i=0}^{k-1} \frac{a_{i} a_{k-1-i} \Gamma(1+(k-1) \alpha)}{\Gamma(1+i \alpha) \Gamma(1+(k-1-i) \alpha)}\right)\right. \\
& \left.-\left(\sum_{i=0}^{k-2} \frac{a_{i} a_{k-2-i} \Gamma(1+(k-1) \alpha)}{\Gamma(1+i \alpha) \Gamma(1+(k-1-i) \alpha)}\right)\right]
\end{aligned}
$$

for $k=1,2,3, \ldots$

Proof. Let

$$
u_{k}(t)=a_{0}+\sum_{n=1}^{k} \frac{a_{n} t^{n \alpha}}{\Gamma(1+n \alpha)}
$$

be the approximate solution of Equation (5) subject to the initial condition (6). Then the $k$ th residual function is expressed as

$$
\begin{aligned}
\operatorname{Res}_{k}(t)= & \kappa D_{t}^{\alpha} u_{k}(t)-u_{k}(t)+u_{k}^{2}(t)+u_{k}(t) I^{\alpha}\left(u_{k}(t)\right) \\
= & \kappa D_{t}^{\alpha}\left(\sum_{n=0}^{k} \frac{a_{n} t^{n \alpha}}{\Gamma(1+n \alpha)}\right)-\left(\sum_{n=0}^{k} \frac{a_{n} t^{n \alpha}}{\Gamma(1+n \alpha)}\right) \\
& {\left[\sum_{n=0}^{k}\left(\sum_{i=0}^{k} \frac{a_{i} a_{n-i}}{\Gamma(1+i \alpha) \Gamma(1+(n-i) \alpha)}\right) t^{n \alpha}\right.} \\
& \left.+\sum_{n=1}^{k}\left(\sum_{i=n}^{k} \frac{a_{i} a_{k+n-i}}{\Gamma(1+i \alpha) \Gamma(1+(k+n-i) \alpha)}\right) t^{(k+n) \alpha}\right] \\
& +\left(\sum_{n=0}^{k} \frac{a_{n} t^{n \alpha}}{\Gamma(1+n \alpha)}\right) I^{\alpha}\left(\sum_{n=0}^{k} \frac{a_{n} t^{n \alpha}}{\Gamma(1+n \alpha)}\right) .
\end{aligned}
$$

So,

$$
\begin{aligned}
\operatorname{Res}_{k}(t)= & \kappa \sum_{n=1}^{k} \frac{a_{n} t^{(n-1) \alpha}}{\Gamma(1+(n-1) \alpha)}-\left(\sum_{n=0}^{k} \frac{a_{n} t^{n \alpha}}{\Gamma(1+n \alpha)}\right) \\
& {\left[\sum_{n=0}^{k}\left(\sum_{i=0}^{k} \frac{a_{i} a_{n-i}}{\Gamma(1+i \alpha) \Gamma(1+(n-i) \alpha)}\right) t^{n \alpha}\right.} \\
& \left.+\sum_{n=1}^{k}\left(\sum_{i=n}^{k} \frac{a_{i} a_{k+n-i}}{\Gamma(1+i \alpha) \Gamma(1+(k+n-i) \alpha)}\right) t^{(k+n) \alpha}\right] \\
& \left.+\left[\sum_{n=0}^{k}\left(\sum_{i=0}^{k} \frac{a_{i} a_{n-i}}{\Gamma(1+i \alpha) \Gamma(1+(n+1-i) \alpha)}\right) t^{(n+1) \alpha}\right) t^{(k+n+1) \alpha}\right] \\
& +\sum_{n=1}^{k}\left(\sum_{i=n}^{k} \frac{a_{i} a_{k+n-i}}{\Gamma(1+i \alpha) \Gamma(1+(k+n+1-i) \alpha)}\right]
\end{aligned}
$$


Now, operating $D_{t}^{(k-1) \alpha}$ on both sides of Equation (27) yields

$$
\begin{aligned}
D_{t}^{(k-1) \alpha} \operatorname{Res}_{k}(t)= & \kappa a_{k}-\left(a_{k-1}+\frac{a_{k} t^{\alpha}}{\Gamma(1+\alpha)}\right) \\
& +\left[\sum_{i=0}^{k-1} \frac{a_{i} a_{k-1-i} \Gamma(1+(k-1) \alpha)}{\Gamma(1+i \alpha) \Gamma(1+(k-1-i) \alpha)}\right. \\
& +\sum_{i=0}^{k} \frac{a_{i} a_{k-i} \Gamma(1+k \alpha)}{\Gamma(1+i \alpha) \Gamma(1+(k-i) \alpha) \Gamma(1+\alpha)} t^{\alpha} \\
& \left.+\sum_{n=1}^{k}\left(\sum_{i=n}^{k} \frac{a_{i} a_{k+n-i} \Gamma(1+(k+n) \alpha)}{\Gamma(1+i \alpha) \Gamma(1+(k+n-i) \alpha) \Gamma(1+(n+1) \alpha)}\right) t^{(n+1) \alpha}\right] \\
& +\left[\sum_{i=0}^{k-2} \frac{a_{i} a_{k-2-i} \Gamma(1+(k-1) \alpha)}{\Gamma(1+i \alpha) \Gamma(1+(k-1-i) \alpha)}\right. \\
& +\sum_{i=0}^{k-1} \frac{a_{i} a_{k-1-i} \Gamma(1+k \alpha)}{\Gamma(1+i \alpha) \Gamma(1+(k-i) \alpha) \Gamma(1+\alpha)} t^{\alpha} \\
& +\sum_{i=0}^{k} \frac{a_{i} a_{k-i} \Gamma(1+(k+1) \alpha)}{\Gamma(1+i \alpha) \Gamma(1+(k+1-i) \alpha) \Gamma(1+2 \alpha)} t^{2 \alpha} \\
& \left.+\sum_{n=1}^{k}\left(\sum_{i=n}^{k} \frac{a_{i} a_{k+n-i} \Gamma(1+(k+n+1) \alpha)}{\Gamma(1+i \alpha) \Gamma(1+(k+n+1-i) \alpha) \Gamma(1+(n+2) \alpha)}\right) t^{(n+2) \alpha}\right] .
\end{aligned}
$$

Then, we solve $D_{t}^{(k-1) \alpha} \operatorname{Res}_{k}(0)=0$ to obtain

$$
\begin{aligned}
a_{k}= & \frac{1}{\mathcal{\kappa}}\left[a_{k-1}-\left(\sum_{i=0}^{k-1} \frac{a_{i} a_{k-1-i} \Gamma(1+(k-1) \alpha)}{\Gamma(1+i \alpha) \Gamma(1+(k-1-i) \alpha)}\right)\right. \\
& \left.-\left(\sum_{i=0}^{k-2} \frac{a_{i} a_{k-2-i} \Gamma(1+(k-1) \alpha)}{\Gamma(1+i \alpha) \Gamma(1+(k-1-i) \alpha)}\right)\right] .
\end{aligned}
$$

\section{Convergence Analysis}

Now, we prove the convergence of the MRPSM. We start by Lemma 1 which is a connection between the classical power series (CPS) and the fractional power series.

Lemma 1. The classical power series $\sum_{n=0}^{\infty} u_{n} t^{n},-\infty<t<\infty$, has a radius of convergence $R$ if and only if the fractional power series $\sum_{n=0}^{\infty} a_{n} t^{n \alpha}, t \geq 0$, has a radius of convergence $R^{\frac{1}{\alpha}}$.

Proof. See [38].

The next theorem indicates that the series solution of nonlinear fractional Volterra population growth model converges in a neighborhood of $t=0$.

Theorem 5. The fractional power series solution of the nonlinear fractional Volterra population growth model (5) subject to the initial condition (6)

$$
u(t)=\sum_{n=0}^{\infty} \frac{a_{n} t^{n \alpha}}{\Gamma(1+n \alpha)}
$$

where $a_{n}$ are the coefficients in Equation (26), has a positive radius of convergence. 
Proof. From Equation (26), we can see that

$$
\begin{aligned}
\frac{\left|a_{k}\right|}{\Gamma(1+k \alpha)} \leq & \left|\frac{1}{\kappa}\right| \frac{\left|a_{k-1}\right|}{\Gamma(1+k \alpha)}+\left|\frac{1}{\kappa}\right| \frac{\left|\sum_{i=0}^{k-1} \frac{a_{i} a_{k-1-i} \Gamma(1+(k-1) \alpha)}{\Gamma(1+i \alpha) \Gamma(1+(k-1-i) \alpha)}\right|}{\Gamma(1+k \alpha)} \\
& +\left|\frac{1}{\mathcal{\kappa}}\right| \frac{\left|\sum_{i=0}^{k-2} \frac{a_{i} a_{k-2-i} \Gamma(1+(k-1) \alpha)}{\Gamma(1+i \alpha) \Gamma(1+(k-1-i) \alpha)}\right|}{\Gamma(1+k \alpha)} \\
\leq & \left|\frac{1}{\kappa}\right| \frac{\left|a_{k-1}\right|}{\Gamma(1+k \alpha)} \\
& +\left|\frac{1}{\mathcal{\kappa}}\right| \max _{0 \leq i \leq k-1}\left\{\frac{\Gamma(1+(k-1) \alpha)}{\Gamma(1+i \alpha) \Gamma(1+(k-1-i) \alpha) \Gamma(1+k \alpha)}\right\} \sum_{i=0}^{k-1}\left|a_{i}\right|\left|a_{k-1-i}\right| \\
& +\left|\frac{1}{\mathcal{\kappa}}\right| \max _{0 \leq i \leq k-2}^{k-2}\left\{\frac{\Gamma(1+(k-1) \alpha)}{\Gamma(1+i \alpha) \Gamma(1+(k-1-i) \alpha) \Gamma(1+k \alpha)}\right\} \sum_{i=0}^{k-2}\left|a_{i}\right|\left|a_{k-2-i}\right| \\
= & A\left|a_{k-1}\right|+B \sum_{i=0}^{k-1}\left|a_{i}\right|\left|a_{k-1-i}\right|+C \sum_{i=0}^{k}\left|a_{i}\right|\left|a_{k-2-i}\right|,
\end{aligned}
$$

where

$$
\begin{aligned}
& A=\left|\frac{1}{\kappa}\right| \Gamma(1+k \alpha), B=\max _{0 \leq i \leq k-1}\left\{\frac{\Gamma(1+(k-1) \alpha)}{\Gamma(1+i \alpha) \Gamma(1+(k-1-i) \alpha) \Gamma(1+k \alpha)}\right\}\left|\frac{1}{\kappa}\right| \\
& C=\max _{0 \leq i \leq k-2}\left\{\frac{\Gamma(1+(k-1) \alpha)}{\Gamma(1+i \alpha) \Gamma(1+(k-1-i) \alpha) \Gamma(1+k \alpha)}\right\}\left|\frac{1}{\kappa}\right| .
\end{aligned}
$$

Let

$$
f(t)=\sum_{k=0}^{\infty} b_{k} t^{k}
$$

where $b_{0}=\left|a_{0}\right|, b_{1}=\frac{\left|a_{1}\right|}{\Gamma(1+\alpha)}$ and

$$
b_{k}=A b_{k-1}+B \sum_{i=0}^{k-1} b_{i} b_{k-1-i}+C \sum_{i=0}^{k-2} b_{i} b_{k-2-i}, k=2,3,4, \ldots
$$

be the classical power series. Thus,

$$
\begin{aligned}
\omega=f(t) & =b_{0}+b_{1} t+\sum_{k=2}^{\infty} b_{k} t^{k} \\
& =b_{0}+b_{1} t+A \sum_{k=2}^{\infty} b_{k-1} t^{k}+B \sum_{k=2}^{\infty}\left(\sum_{i=0}^{k-1} b_{i} b_{k-1-i}\right) t^{k}+C \sum_{k=2}^{\infty}\left(\sum_{i=0}^{k-2} b_{i} b_{k-2-i}\right) t^{k} \\
& =b_{0}+b_{1} t+A t \sum_{k=1}^{\infty} b_{k} t^{k}+B t \sum_{k=1}^{\infty}\left(\sum_{i=0}^{k} b_{i} b_{k-i}\right) t^{k}+C t^{2} \sum_{k=0}^{\infty}\left(\sum_{i=0}^{k} b_{i} b_{k-i}\right) t^{k} .
\end{aligned}
$$

Let

$$
G(t, \omega)=\omega-b_{0}-b_{1} t-A t\left(\omega-b_{0}\right)-B t\left(\omega^{2}-b_{0}^{2}\right)-C t^{2} \omega^{2}
$$

Then

$$
G_{\omega}(t, \omega)=1-t A-2 B t \omega-2 C t^{2} \omega .
$$

Regarding at point $\left(0, b_{0}\right)$, the function $G(t, \omega)$ is 0 and the partial derivative of the function $G(t, \omega)$ with respect to $\omega$ is 1 . We can see that $G(t, \omega)$ is an analytic function, so $G(t, \omega)$ has continuous derivatives. By implicit function theorem [40], there is a neighborhood of $\left(0, b_{0}\right)$ so 
that whenever $t$ is sufficiently close to 0 there is a unique $\omega$ so that $G(t, \omega)=0$. Then, $f(t)$ is an analytic function in the neighborhood of the point $\left(0, b_{0}\right)$ of the $(t, \omega)$-plane with a positive radius of convergence. From Lemma 1, the series in Equation (7) converges. The proof is complete.

\section{Numerical Example}

In this section, a numerical example of the MRPSM for nonlinear fractional Volterra population growth model is presented.

Consider the following nonlinear fractional Volterra population growth model

$$
\kappa D_{t}^{\alpha} u(t)=u(t)-u^{2}(t)-u(t) I^{\alpha} u(t), \alpha \in(0,1],
$$

subject to

$$
u(0)=0.4 \text {. }
$$

The graphical results of the solution for Equation (31) and initial condition (32) is illustrated through Figures 1-3 for different $\alpha$ and $\kappa$ values. Figure 1 represents the behavior of an approximate solution $\kappa=0.7$ with various values of $\alpha$. Figures 2 and 3 show the behavior of an approximate solution for $\alpha=0.75$ and 1 with various values of $\kappa$. We observe that increasing the parameter $\kappa$ resulted in decreasing of the approximate solution.

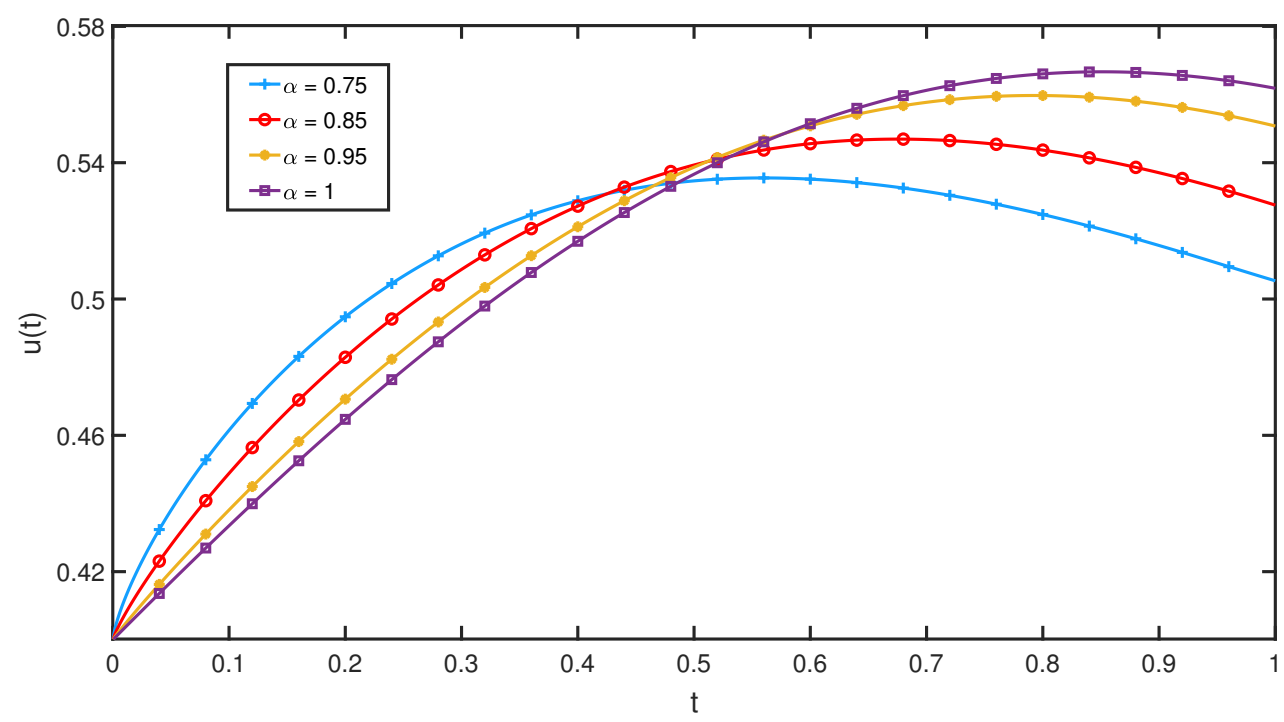

Figure 1. Approximate solution of Equation (31) for various of $\alpha$ and $\kappa=0.7$.

For $\alpha=1$, Equation (31) becomes the classical Volterra population growth model. Table 1 represents the relation between $\kappa, u_{\max }$ and $t_{\text {critical }}$. The maximum value is presented as $u_{\max }$ and the position of $u_{\max }$ is presented as $t_{\text {critical }}$. The exact value of $u_{\max }$ was evaluated by using

$$
u_{\max }=1+\kappa \ln \left(\frac{\kappa}{1+\kappa-u(0)}\right)
$$

obtained by [32].

In Table 1, we observe that the approximate value of $u_{\max }$ decreases as $\kappa$ increases. It is noted that the approximate value of $u_{\max }$ is close to the exact value of $u_{\max }$ for all values of $\kappa$. In fact, the results reported in Table 1 illustrates the validity and good accuracy of the method.

In order to show the convergence of the MRPSM, the absolute errors of $u_{\max }$ for different $\kappa$ values and $\alpha=1$ are shown in Table 2. The absolute error tends to decrease when the number of truncated terms $(k)$ increases. This shows that the method works reasonably for the classical problem. 
The results for other values of $\alpha$ are shown in Table 3. The approximate solution of $u_{\max }$ at each $\alpha$ and $\kappa$ changes slightly when the number of truncated terms increases. We can say that only 20-term approximation is acceptable to explain the behavior of the population with less computational effort. Table 4 demonstrates how the step size $h$ affects the approximation for $\alpha=1$. Compare to the exact values of $u_{\max }$ in Table 1 , it is natural that the smaller step size is, the better approximation performs. For $h=0.002$, the computation time is less than 0.08 second which we hardly have to wait.

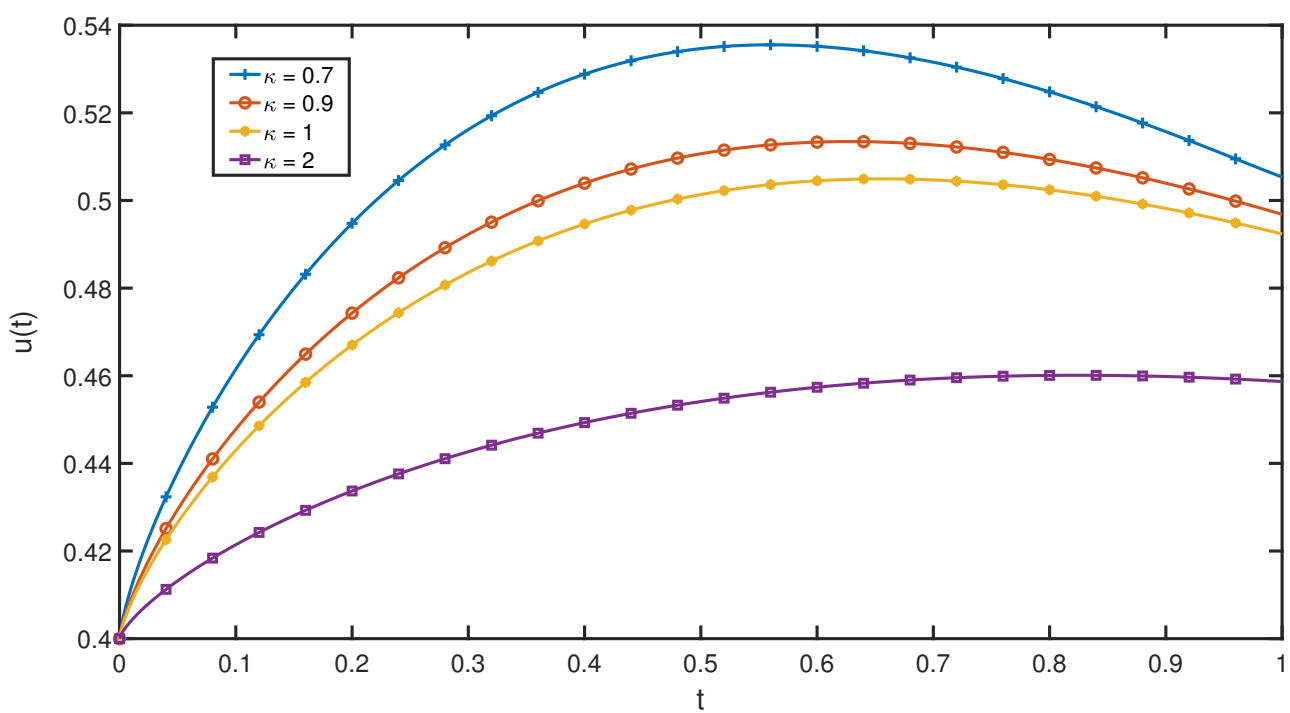

Figure 2. Approximate solution of Equation (31) for various of $\kappa$ and $\alpha=0.75$.

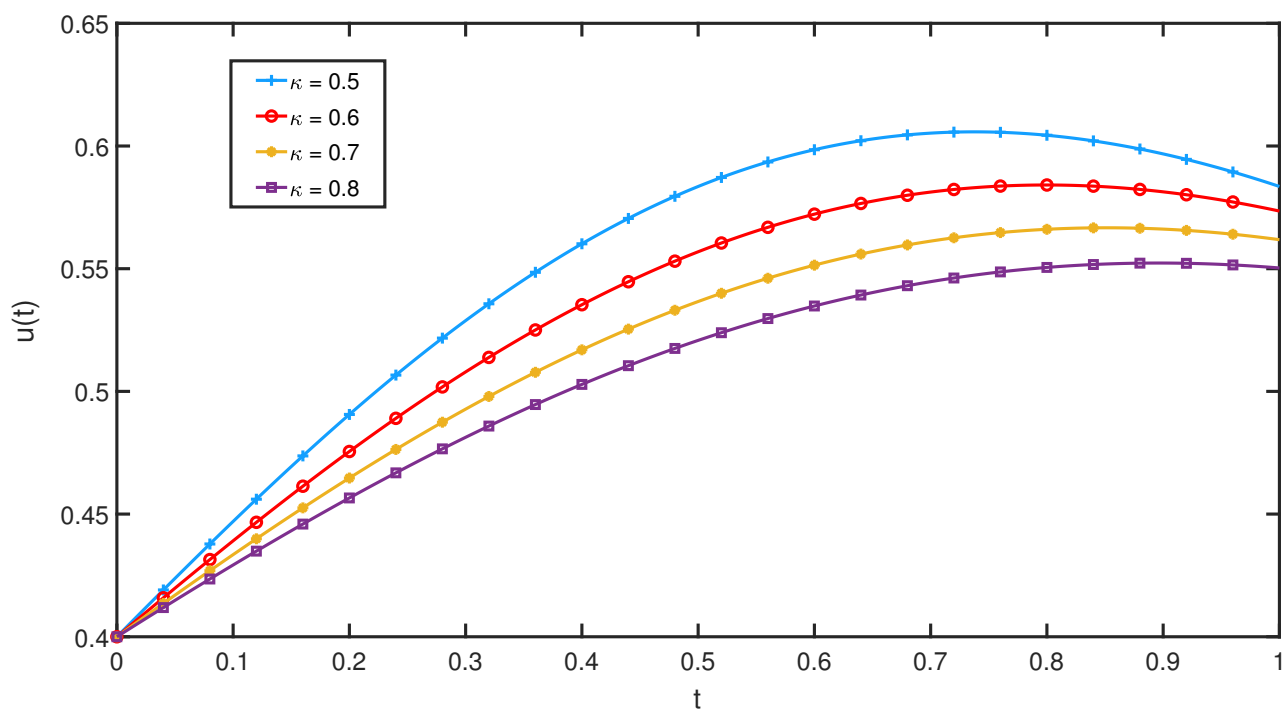

Figure 3. Approximate solution of Equation (31) for various of $\kappa$ and $\alpha=1$.

Table 1. The approximation of $u_{\max }$ and exact value of $u_{\max }$ for various of $\kappa$ at $\alpha=1$.

\begin{tabular}{ccccc}
\hline $\boldsymbol{\kappa}$ & $\boldsymbol{t}_{\text {critical }}$ & Approximate $\boldsymbol{u}_{\max }$ & Exact $\boldsymbol{u}_{\max }$ & Absolute Errors \\
\hline 0.5 & 0.738 & 0.6057712361 & 0.6057713198 & $8.37213 \times 10^{-8}$ \\
0.6 & 0.800 & 0.5841115047 & 0.5841116917 & $1.86934 \times 10^{-7}$ \\
0.7 & 0.852 & 0.5666724313 & 0.5666725541 & $1.22786 \times 10^{-7}$ \\
0.8 & 0.896 & 0.5523073685 & 0.5523073697 & $1.14278 \times 10^{-9}$ \\
\hline
\end{tabular}


Table 2. Absolute error of $u_{\max }$ for various of $\kappa$ and $\alpha=1$.

\begin{tabular}{cccc}
\hline $\boldsymbol{\kappa}$ & $\boldsymbol{k}=\mathbf{2 0}$ & $\boldsymbol{k}=\mathbf{2 5}$ & $\boldsymbol{k}=\mathbf{3 0}$ \\
\hline 0.5 & $3.33491 \times 10^{-6}$ & $4.48341 \times 10^{-7}$ & $8.37213 \times 10^{-8}$ \\
0.6 & $1.12750 \times 10^{-6}$ & $2.47310 \times 10^{-7}$ & $1.86934 \times 10^{-7}$ \\
0.7 & $4.28738 \times 10^{-7}$ & $1.34831 \times 10^{-7}$ & $1.22786 \times 10^{-7}$ \\
0.8 & $1.10182 \times 10^{-7}$ & $3.89890 \times 10^{-9}$ & $1.14278 \times 10^{-9}$ \\
\hline
\end{tabular}

Table 3. The approximation of $u_{\max }$ for different $\alpha$ and $\kappa$ values.

\begin{tabular}{ccccc}
\hline $\boldsymbol{\alpha}$ & $\boldsymbol{\kappa}$ & $\boldsymbol{k}=\mathbf{2 0}$ & $\boldsymbol{k}=\mathbf{2 5}$ & $\boldsymbol{k}=\mathbf{3 0}$ \\
\hline 0.75 & 0.6 & 0.5502368550 & 0.5502415323 & 0.5502426850 \\
0.85 & 0.7 & 0.5469171943 & 0.5469180381 & 0.5469181062 \\
0.95 & 0.8 & 0.5458638855 & 0.5458640377 & 0.5458640426 \\
\hline
\end{tabular}

Table 4. The approximation of $u_{\max }$ and computation time for $\alpha=1$ and $k=20$.

\begin{tabular}{ccccc}
\hline \multirow{2}{*}{$\boldsymbol{h}$} & \multicolumn{2}{c}{$\boldsymbol{\kappa}=\mathbf{0 . 5}$} & \multicolumn{2}{c}{$\kappa=\mathbf{0 . 7}$} \\
\cline { 2 - 5 } & Approximate $\boldsymbol{u}_{\text {max }}$ & Computation Time (s) & Approximate $\boldsymbol{u}_{\text {max }}$ & Computation Time (s) \\
\hline 0.002 & 0.605771236096562 & 0.078953 & 0.566672431329788 & 0.075677 \\
0.02 & 0.605769084446570 & 0.072933 & 0.566655106763389 & 0.070760 \\
0.1 & 0.605246758144338 & 0.070844 & 0.566135276851748 & 0.067380 \\
\hline
\end{tabular}

\section{Conclusions}

In this paper, we proposed a computational method called the modified residual power series method (MRPSM) for solving nonlinear fractional Volterra population growth model. A closed form of the fractional power series solution is obtained which is the advantage of this method. The convergence analysis was also investigated. We gave a numerical example supporting that this method is efficiently applicable for the nonlinear fractional Volterra population growth model with high accuracy. Finally, it can easily be applied to other fractional nonlinear initial value problems to obtain numerical or analytical solutions.

Author Contributions: Conceptualization, P.D. and D.P.; methodology, P.D.; validation, P.D. and D.P.; formal analysis, A.W. and P.D.; investigation, D.P.; writing-original draft preparation, P.D. and D.P.; writing-review and editing, A.W. and P.D. All authors have read and agreed to the published version of the manuscript.

Funding: This research received no external funding.

Acknowledgments: The authors are grateful to the referees for their careful reading of the manuscript and their useful comments.

Conflicts of Interest: The authors declare no conflict of interest.

\section{Abbreviations}

The following abbreviations are used in this manuscript:

RPSM residual power series method

ADM Adomain decomposition method

RPS residual power series

MRPSM modified residual power series method

FPS fractional power series

CPS classical power series 


\section{References}

1. Debnath, L. Recent application of fractional calculus to science and engineering. Int. J. Math. Math. Sci. 2003, 3413-3442. [CrossRef]

2. Bohannan, G.W. Analog fractional order controller in temperature and motor control applications. J. Vib. Contr. 2008, 14, 1487-1498. [CrossRef]

3. Panda, R.; Dash, M. Fractional generalized splines and signal processing. Signal Process 2006, 14, $2340-2350$. [CrossRef]

4. Baillie, R.T. Long memory processes and fractional integration in econometrics. J. Economet. 1996, 73, 5-59. [CrossRef]

5. Bagley, R.L.; Torvik, P.J. A theoretical basis for the application of fractional calculus to viscoelasticity. J. Rheol. 1983, 27, 201-210. [CrossRef]

6. Yang, X.J.; Gao, F.; Yang, J. General Fractional Derivatives with Applications in Viscoelasticity; Academic Press (Elsevier Science Publishers): Heidelberg, Geremany; London, UK; New York, NY, USA; Amsterdam, The Netherlands, 2020.

7. Yang, X.J. Local Fractional Integral Transforms and Their Applications; Academic Press (Elsevier Science Publishers): Heidelberg, Geremany; London, UK; New York, NY, USA; Amsterdam, The Netherlands, 2016.

8. Chow, T.S. Fractional dynamics of interfaces between soft nanoparticles and rough substrates. Phys. Lett. A 2005, 342, 148-155. [CrossRef]

9. Mainardi, F. Fractional calculus: Some basic problems in continuum and statistical mechanics. Fractals Fract. Calc. Contin. Mech. 1997, 378, 291-348.

10. Yang, X.J.; Gao, F.; Yang, J.; Zhou, H.W. Fundamental solutions of the general fractional-order diffusion equations. Math. Methods Appl. Sci. 2018, 18, 9312-9320. [CrossRef]

11. Yang, X.J.; Gao, F.; Srivastava, H.M. Non-differentiable exact solutions for the nonlinear ODEs defined on fractal sets. Fractals 2017, 25, 1740002. [CrossRef]

12. Ganji, R.M.; Jafari, H.A. New approach for solving nonlinear volterra integro-differential equations with Mittag-Leffler kernel. Proc. Inst. Math. Mech. 2020, 46, 144-158.

13. Jafari, H.; Tajadodi, H.; Baleanu, D. Numerical solution of variable order integro-differential equations. Adv. Math. Model. Appl. 2019, 4, 64-69.

14. Jafari, H.; Seifi, S. Homotopy analysis method for solving linear and nonlinear fractional diffusion-wave equation. Commun. Nonlinear Sci. 2009, 14, 2006-2012. [CrossRef]

15. He, J.H. Homotopy perturbation technique. Comput. Methods Appl. Mech. Eng. 1999, 178, 257-262. [CrossRef]

16. Jafari, H.; Momani, S. Solving fractional diffusion and wave equations by modified homotopy perturbation method. Phys. Lett. A 2007, 370, 388-396. [CrossRef]

17. Tiwana, M.H.; Maqbool, K.; Mann, A.B. Homotopy perturbation Laplace transform solution of fractional non-linear reaction diffusion system of Lotka-Volterra type differential equation. Eng. Sci. Technol. Int. J. 2017, 20, 672-678. [CrossRef]

18. Kumar, D.; Singh, J.; Kumar, S. Numerical computation of fractional multidimensional diffusion equations by using a modified homotopy perturbation method. J. Assoc. Arab Univ. Basic Appl. Sci. 2015, 17, 20-26.

19. Singh, B.K.; Srivastava, V.K. Approximate series solution of multi-dimensional, time fractional-order (heatlike) diffusion equations using FRDTM. R. Soc. Open Sci. 2018, 2, 140-511.

20. Jafari, H.; Gejji, V. Solving linear and nonlinear fractional diffusion and wave equations by adomian decomposition. J. Appl. Math. Comput. 2006, 180, 488-497. [CrossRef]

21. Ganji, D.D.; Tari, H.; Jooybari, M.B. Variational iteration method and homotopy perturbation method for nonlinear evolution equations. Comput. Math. Appl. 2007, 54, 1018-1027. [CrossRef]

22. Wazwaz, A.M. The variational iteration method for rational solutions for $\mathrm{K}(2,2)$, Burgers, and cubic Boussinesq equations. J. Comput. Appl. Math. 2007, 207, 18-23. [CrossRef]

23. Arqub, A.O. Series solution of fuzzy differential equations under strongly generalized differentiability. J. Adv. Res. Appl. Math. 2013, 5, 31-52. [CrossRef]

24. Alquran, M. Analytical solutions of fractional foam drainage equation by residual power series method. Math. Sci. 2014, 8, 153-160. [CrossRef]

25. Alshammari, S.; Alsamadi, M.; Alshammari, M.; Hashim, M.I.; Alias, M.A. Advanced analytical treatment of fractional logistic equations based on residual error functions. Int. J. Differ. Equ. 2019, 2019, 1-11. [CrossRef] 
26. Chen, B.; Qin, L.; Xu, F.; Zu, J. Applications of general residual power series method to differential equations with variable coefficients. Discrete Dyn. Nat. Soc. 2018, 2018, 1-9. [CrossRef]

27. Kumar, A.; Kumar, S.; Singh, M. Residual power series method for fractional Sharma-Tasso-Olever equation. Commun. Numer. Anal. 2016, 1, 1-10. [CrossRef]

28. Prakasha, D.G.; Veeresha, P.; Baskonus, H.M. Residual power series method for fractional Swift-Hohenberg equation. Fractal Fract. 2019, 3, 9. [CrossRef]

29. Tariq, H.; Akram, G. Residual power series method for solving time-space-fractional Benney-Lin equation arising in falling film problems. J. Appl. Math. Comput. 2017, 55, 683-708. [CrossRef]

30. Zhang, Y.; Kumar, A.; Kumar, S.; Baleanu, D.; Yang, X. Residual power series method for time-fractional Schrödinger equations. J. Nonlinear Sci. Appl. 2016, 9, 5821-5829. [CrossRef]

31. Scudo, F.M. Vito Volterra and theoretical ecology. Theor. Popul. Biol. 1971, 2, 1-23. [CrossRef]

32. TeBeest, K.G. Classroom note: Numerical and analytical solutions of Volterra's population Model. Soc. Ind. Appl. Math. 1997, 39, 484-493.

33. Adomian, G. A review of the decomposition method in applied mathematics. J. Math. Anal. Appl. 1988, 135, 501-544. [CrossRef]

34. Eltayeb, H.; Abdalla, Y.T.; Bachar, I.; Khabir, M.H. Fractional telegraph equation and its solution by natural transform decomposition method. Symmetry 2019, 11, 334. [CrossRef]

35. Eltayeb, H.; Bachar, I.; Kilicman, A. On conformable double Laplace transform and one dimensional fractional coupled Burger's equation. Symmetry 2019, 11, 417. [CrossRef]

36. Al-Khaled, K.; Hazaimeh, A. Comparison method for solving non-linear Sturm-Liouville eigenvalues problems. Symmetry 2020, 12, 1179. [CrossRef]

37. Podlubny, I. Fractional Differential Equations; Academic Press: San Diego, CA, USA, 1999.

38. El-Ajou, A.E.; Arqub, O.A.; Zhour, Z.A.; Momani, S. New results on fractional power series: Theories and applications. Entropy 2013, 15, 5305-5323. [CrossRef]

39. Biazar, J.; Shafiof, S.M. A simple algorithm for calculating Adomian polynomials. Int. J. Contemp. Math. Sci. 2007, 2, 975-982. [CrossRef]

40. Rudin, W. Principles of Mathematical Analysis, 3rd ed.; China Machine Press: Beijing, China, 2004.

Publisher's Note: MDPI stays neutral with regard to jurisdictional claims in published maps and institutional affiliations.

(C) 2020 by the authors. Licensee MDPI, Basel, Switzerland. This article is an open access article distributed under the terms and conditions of the Creative Commons Attribution (CC BY) license (http://creativecommons.org/licenses/by/4.0/). 